\title{
Evaluation of the interrupter technique in healthy, unsedated infants
}

\author{
G.L. Hall, J.H. Wildhaber, M. Cernelc, U. Frey
}

Evaluation of the interrupter technique in healthy, unsedated infants. G.L. Hall, J.H. Wildhaber, M. Cernelc, U. Frey. C) ERS Journals Ltd 2001.

ABSTRACT: The interrupter technique may be used to monitor respiratory resistance and does not require active patient cooperation, but has yet to be applied in unsedated, spontaneously breathing infants. The aim of this study was to determine if the interrupter technique is feasible in spontaneously breathing infants and to investigate the influence of facemask types and analysis techniques on the interrupter resistance (Rint).

$R$ int was measured in 14 healthy, unsedated, sleeping infants (aged 38.4 (31-56) days (mean (range)). Paired measurements were made using large volume, compliant (Mcomp) and small volume, rigid (Mrigid) facemasks. Flow and pressure were measured at the airway opening prior to- and following a brief airway occlusion $(500 \mathrm{~ms})$. Rint was calculated using four previously reported analysis techniques.

$R$ int could be measured in all infants. Mcomp, independent of the analysis method significantly underestimated $R$ int $(p<0.001)$. The variability and magnitude of $R$ int were significantly influenced by the choice of analysis method.

The conclusion is that the interrupter technique is feasible in spontaneously breathing, unsedated infants. Equipment design and analysis method significantly influences interrupter resistance. Studies standardizing equipment and identifying the most appropriate analysis technique in this age group are needed.

Eur Respir J 2001; 18: 982-988.
Swiss Paediatric Respiratory Physiology Research Group, University Children's Hospital, Berne \& Zürich, Switzerland.

Correspondence: U. Frey, Paediatric Respiratory Medicine, Dept of Paediatrics, University Hospital, Inselspital, Berne CH-3010, Switzerland.

Fax: 41316329484

Keywords: Airway resistance, interrupter technique, respiratory resistance, unsedated infants

Received: March 62001

Accepted after revision August 102001

This work was supported by the Swiss National Science Foundation (Score grant: 3200-052197.97/1), the National Health and Medical Research Council of Australia, the Swiss Cystic Fibrosis Society and AstraZeneca (Switzerland).
The interrupter technique is one of the classical methods for determining the Newtonian resistance of the airway tree. While the technique has some limitations, it only requires minimal patient cooperation hence is of particular interest in young children and infants. There have been a number of recent studies conducted in spontaneously breathing children as young as two years. In particular, the application of this technique to pre-school children has allowed an improved understanding of respiratory disease in this age group [1-4]. The interrupter technique, however, is yet to be standardized and has not been applied to spontaneously breathing infants. The interrupter technique involves rapidly occluding the airway opening and assumes that alveolar pressure $(P$ alv $)$ equilibrates rapidly with airway opening pressure $(P$ ao $)$, following the occlusion, hence allowing the determination of $P$ alv. Following flow interruption of the airway, two distinct phases are seen in the $P$ ao trace. There is an initial rapid rise, followed by a secondary, slower rise to a plateau. The work of BATES and co-workers $[5,6]$ in animal models has provided a better understanding of the physiological meaning of these changes. The initial rapid rise in $P$ ao reflects the resistive drop across the airway tree and the initial part of the resistive drop across the respiratory tissues, including the chest wall. The slower, secondary change reflects the stress relaxation of the respiratory tissues and to a lesser extent commuting air. The pre-occlusion flow $\left(V^{\prime}\right)$ and post-occlusion change in $P$ ao can then be used to calculate the interrupter resistance (Rint). Pao following $V^{\prime}$ interruption is influenced by the closure time of the occlusion valve and the compliance of the upper airways (Cua) [7-9]. The analysis method of the preand post-occlusion $V^{\prime}$ and $P$ ao can also influence the magnitude and variability of $\operatorname{Rint}[2,10]$.

Optimal triggering flows ( $V^{\prime}$ int) and occlusion times (tint) may change with differing age groups. Triggering flows, ranging from mid-tidal expiratory flows $(0.2-$ $\left.0.4 \mathrm{~L} \cdot \mathrm{s}^{-1}\right)$ in adults [10] to peak expiratory flow $(0.15-$ $0.2 \mathrm{~L} \cdot \mathrm{s}^{-1}$ ) in five-yr-old children have been reported [2]. In infants, peak expiratory flows (PEF) will be significantly lower than the $0.15-0.2 \mathrm{~L} \cdot \mathrm{s}^{-1}$ range used in young children and thus $V^{\prime}$ int of this magnitude would not be suitable. Previously, tint of $100 \mathrm{~ms}$ has been most commonly applied, although interruptions of $80 \mathrm{~ms}$ have been reported [11]. In infants tint of $\geqslant 500 \mathrm{~ms}$ are used for the single and multiple occlusion techniques [12].

The aim of the study was to test the feasibility of applying the interrupter technique to spontaneously breathing, unsedated infants. This involved identifying suitable $V^{\prime}$ int and tint, and investigating the most appropriate face mask type and analysis of the $V^{\prime}$ and $P$ ao signals. 


\section{Methods}

\section{Subjects}

Fourteen infants (10 males and four females) were studied during quiet sleep, in a supine position with the head in the midline and slightly extended. Infants were aged 38.4 (31-56) days (mean (range)) and had a body weight and length of $4.7(3.57-5.73) \mathrm{kg}$ and 56.5 $(54.0-59.3) \mathrm{cm}$, respectively. The ethics committee of the University Children's Hospital of Berne approved the study; the parents gave written consent and were generally present during the study.

\section{Study design}

The influence of measurement set-up was studied using two facemasks, one a large volume, compliant walled mask (Mcomp), the other a small volume, rigid walled mask (Mrigid), representing the diversity of masks available. Four previously reported analysis techniques, for the estimation of Rint, were employed, utilising the approach of PHAGOo et al. [2, 10, 13-16].

\section{Equipment}

Measurements were carried out using commercially available infant respiratory function equipment (EcoMedics, Duerten, Switzerland). Volume $(V), V^{\prime}$, and $P$ ao were sampled at $200 \mathrm{~Hz}$ with an accuracy of 12 bits. Interruptions were performed with a slide valve (US2000: EcoMedics, Duerten, Switzerland) with a mean closure time of $7.5 \mathrm{~ms}$ (range: 6-9 ms). Based on examination of tidal breathing, interruptions were performed at end inspiration at a triggered expiratory flow of $0.020 \mathrm{~L} \cdot \mathrm{s}^{-1}$, equating to volumes approximating to tidal volume. This flow was chosen to ensure accurate triggering in each infant and to account for the normal variation in end inspiratory flows. Initially, an occlusion time of $100 \mathrm{~ms}$ was employed. However upon inspection of the $P$ ao trace (e.g. fig. 1) it was noted that $P$ ao was rapidly increasing at this time point and as such an accurate confirmation of a relaxed passive respiratory system could not be obtained. The occlusion time was subsequently increased to $500 \mathrm{~ms}$, based on the minimum occlusion time recommended for the single occlusion technique, to ensure that a relaxed passive respiratory system
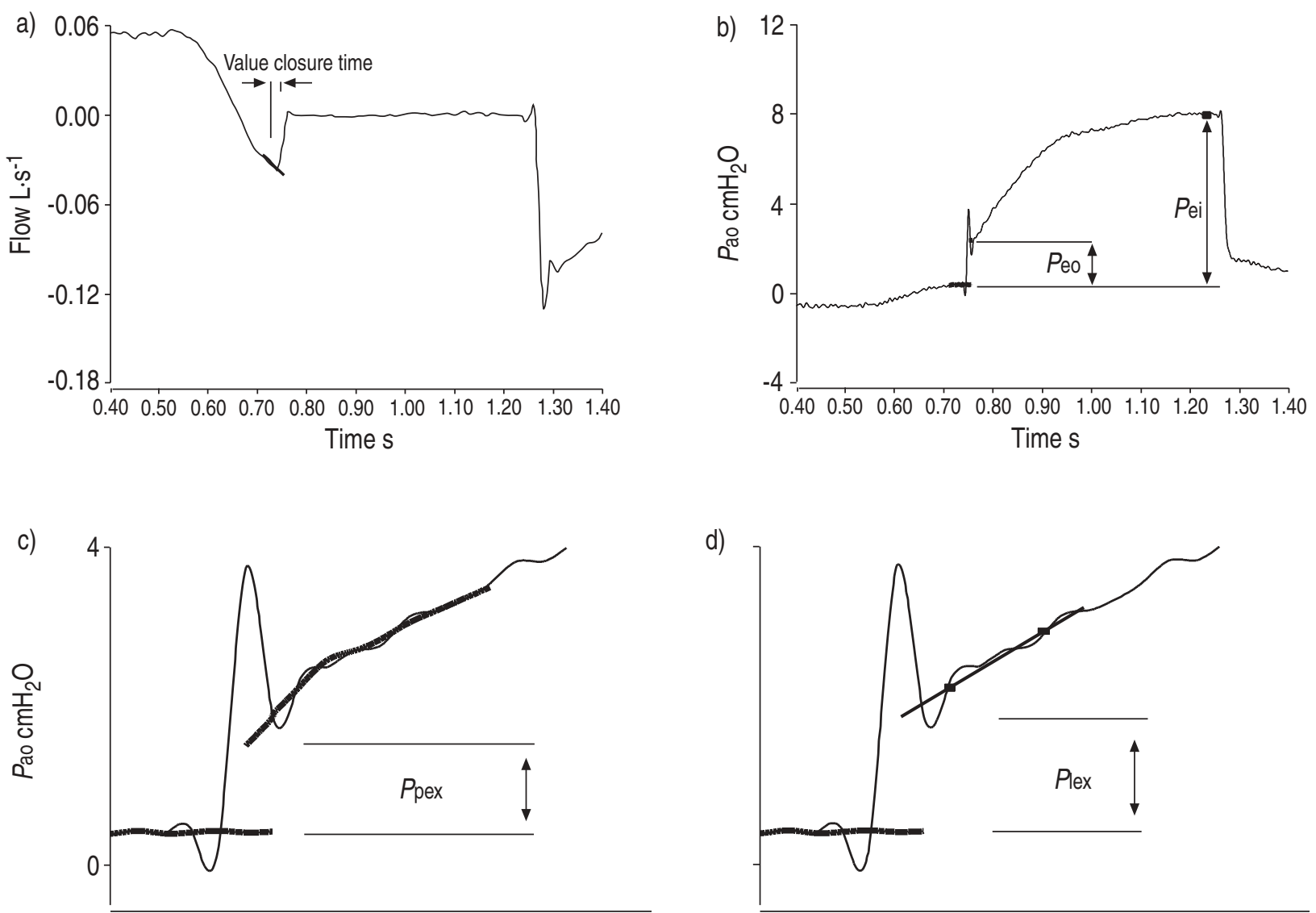

d)

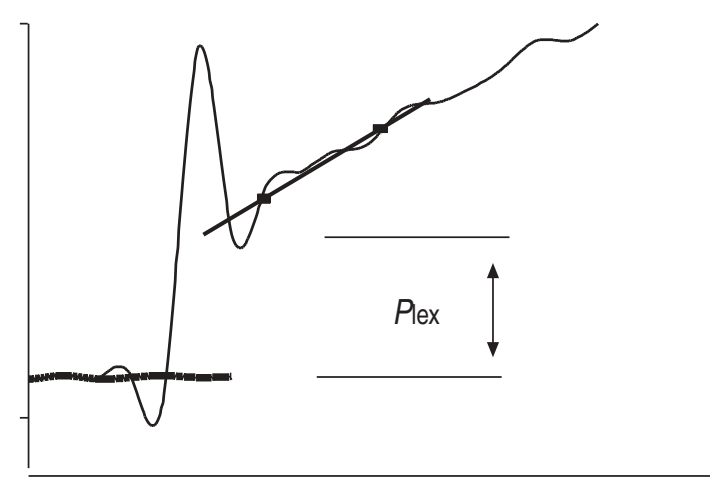

Fig. 1. - a) Representative flow and b) airway opening pressure $(P$ ao $)$ traces following flow interruption in an infant. The interrupter valve was triggered at end inspiration at $20 \mathrm{~mL} \cdot \mathrm{s}^{-1}$ and held closed for $500 \mathrm{~ms}$. The calculation of the pre-occlusion flow ( $\left.V^{\prime}\right)$ and $P$ ao and postocclusion $P$ ao values subsequently used to determine interrupter resistance (Rint) are also illustrated. The initial rapid rise in $P$ ao, followed by a slower, secondary rise to an end-occlusion plateau can be seen. Pre-occlusion $V^{\prime}$ and $P$ ao were calculated by extrapolating a smooth polynomial fitting curve to the time of half valve closure (seen in bold lines). Post-occlusion $P$ ao was determined using four previously published methods. c) $P$ pex was determined by back-extrapolating a smooth fitted curve to a time of half valve closure. d) $P$ lex was the instantaneous $P$ ao at half valve closure obtained from a two-point (centred about 30 and $70 \mathrm{~ms}$ ) linear extrapolation. $P$ eo and $P$ ei were the mean $P$ ao centred about $30 \mathrm{~ms}$ and $475-490 \mathrm{~ms}$ after half valve closure, respectively. 
could be accurately verified [12]. The delay in valve closure due to software and hardware configuration was approximately $40 \mathrm{~ms}$. An ultrasonic flowmeter (M30.8001: EcoMedics, Duerten, Switzerland) was used, and $V$ calculated from the $V^{\prime}$ signal. Pressure was measured using a piezo transducer (M98.350: EcoMedics, Duerten, Switzerland). The dead space of the flow measurement equipment was determined from its physical dimensions and was $3 \mathrm{~mL}$. The compliant facemask (Mcomp: Size 0, Homedica AG, Cham, Switzerland) had total water displacement volume of $20 \mathrm{~mL}$. The smaller, rigid mask (Mrigid: "Silkomed" Size 1: Model: 852713: Jaeger, Hoechberg, Germany) was lined with putty (REP Putty: Magister, Chattanooga. USA) to ensure a leak free fit and to reduce the dead space, which ranged between $2-5 \mathrm{~mL}$. The total effective dead space of the measurement equipment equated to the sum of the flow measurement dead space and the effective dead space of the mask used (50\% of facemask dead space). Hence the equipment dead space was 13 or 4-6 mL for Mcomp and Mrigid, respectively, fulfilling the recently released equipment standards for infant lung function testing [17].

\section{Measurements}

Infants were studied during quiet sleep, with the mask placed over the nose and mouth. The tidal flowvolume loops were examined for leak and to ensure that the infant had adjusted to the additional dead space. Once the infant had stabilized, 20 interruptions were performed over a period of approximately 2 min. This number of interruptions was chosen to ensure that a minimum of five acceptable interruptions would be available for analysis. In initial studies it was found that interrupting every three to four breaths allowed relatively constant interruptions to be applied, without disturbing the infant's sleep patterns and maximized the number of interruptions while minimizing the time required. No correlation was found between Rint magnitude or variability and the time point at which the interruptions were made. This procedure was carried out initially with Mcomp and then repeated using Mrigid. Following collection, data were stored for off-line analysis. Data were examined prior to analysis and excluded if evidence of leak, breathing or incomplete muscle relaxation were noted as follows: an obvious lack of rise in $P$ ao following interruption, or a rapid decay in $P$ ao during interruption, both indicative of leak around the face mask; obvious sudden decreases or increases in $P$ ao during the interruption period resulting from breathing efforts or incomplete relaxation.

\section{Analysis}

Rint was calculated by dividing the change in $P$ ao due to the interruption by the flow just prior to the interruption, and may be given as:

$$
R \text { int }=\mathrm{d} P \text { ao } / V^{\prime} \text { pre }=\left(P_{\text {post }}-P_{\text {pre }}\right) / V^{\prime} \text { pre }
$$

where $P$ pre and $V^{\prime}$ pre equate to the $P$ ao and $V^{\prime}$ prior to interruption and $P$ post is $P$ ao at a given time point following interruption.

$V^{\prime}$ pre and $P$ pre were calculated by fitting a polynomial curve to the $V^{\prime}$ and $P$ ao traces from 150 to $10 \mathrm{~ms}$ prior to the valve closure, the polynomials were then extrapolated from $10 \mathrm{~ms}$ prior to valve closure to the time point when the valve was half closed (tclosure) [15]. Four analysis techniques were used, all previously reported, to ascertain the magnitude of $P$ post (fig. 1). The first three techniques equate to the resistance across the airway tree (i.e. airway resistance), while the fourth technique includes the initial and secondary pressure changes and may be equated to the respiratory system resistance $(R \mathrm{rs})$.

Back extrapolating a fitted smooth curve. A polynomial curve was fitted to the $P$ post data from 30-200 ms after tclosure [15]. This polynomial was then extrapolated to tclosure to obtain $P$ post and Rint determined ( $R$ pex).

Linear back extrapolation. A straight line was fitted through two time points, each being the mean of $10 \mathrm{~ms}$ of data, centred about 30 and $70 \mathrm{~ms}$ following tclosure [14]. The line was the extrapolated to tclosure, $P$ post obtained, and Rint calculated (Rlex).

End-oscillation pressure. The mean pressure 15-25 ms after tclosure was calculated [13]. This was taken to approximate the pressure at the end of the oscillations $(P$ eo $)$ and was used to estimate the interrupter resistance $(R \mathrm{eo})$.

End interruption pressure. The mean pressure at the end of the interruption $P$ ei was obtained (475-490 ms). This pressure includes the initial and secondary pressure changes and the calculated resistance equates to $\operatorname{Rrs}[16]$.

\section{Statistics}

All technically acceptable interruptions were analysed (as detailed above) and the values averaged for each mask separately (mean \pm SD). Group mean data are expressed as mean \pm standard error (SEM). The coefficient of variability $\left(\mathrm{CV}=\mathrm{SD} \cdot \mathrm{mean}^{-1}\right)$ was calculated for each infant and for the group. A two-way analysis of variance (ANOVA) was used to test for the effect of mask type and analysis technique. Pairwise multiple comparisons were carried out using the Student-Newman-Kuels method. The statistical tests used were sufficiently powered to accurately detect significant differences due to the mask or analysis technique (power $>0.8$ ).

\section{Results}

All infants tolerated the measurements well and were not disturbed during the measurement period from their normal sleep. Measurements were normally completed within a $10 \mathrm{~min}$ period following sleep onset. Group PEF ranged $33.1-73.3 \mathrm{~mL} \cdot \mathrm{s}^{-1}(56.3 \pm$ $10.8 \mathrm{~mL} \cdot \mathrm{s}^{-1}$ : mean $\left.\pm \mathrm{SD}\right)$ and the chosen $V^{\prime}$ int of 


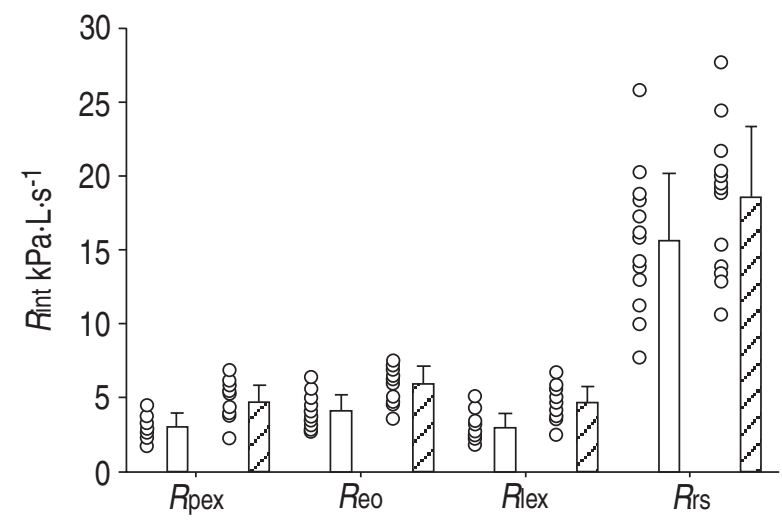

Fig. 2.-Individual $(\bigcirc)$ and group mean (bars (SD) to right of individual data) interrupter resistance (Rint) in healthy, sleeping infants. Rint was significantly underestimated if Mcomp (a compliant, large volume face mask) was used ( $\square$ : Two way ANOVA; $\mathrm{p}<0.001$ ), when compared to Mrigid (a rigid, small volume face mask) $(\mathbb{Z})$. The analysis technique significantly influenced $R$ int (Two way ANOVA: $\mathrm{p}<0.001$ ). Rpex (Rint determined using polynomial back extrapolation method) and $R$ lex (Rint determined using linear back extrapolation method) were significantly lower when compared to Reo (instantaneous Rint following the end of pressure oscillations) (Pairwise comparison: $\mathrm{p}<0.05)$. Rrs: respiratory system resistance.

$20 \mathrm{~mL} \cdot \mathrm{s}^{-1}$ resulted in an interruption success rate of $100 \%$. Group mean inspired volume in the breath immediately prior to occlusion was $30 \pm 1 \mathrm{~mL}$ (mean \pm SEM) and $32 \pm 1 \mathrm{~mL}$, for Mcomp and Mrigid respectively (paired t-test: $\mathrm{p}=0.052$ ), with the volume during interruption being $90.7 \pm 1.6 \%$ and $92.5 \pm 0.4 \%$ of the inspired volume (paired t-test: $\mathrm{p}=0.27$ ). The facemask used proved to have a major influence on Rint. Measurements using Mcomp significantly underestimated Rint, independent of the analysis technique used (fig. 2). Analysis technique significantly influenced Rint, after accounting for the variation due to the

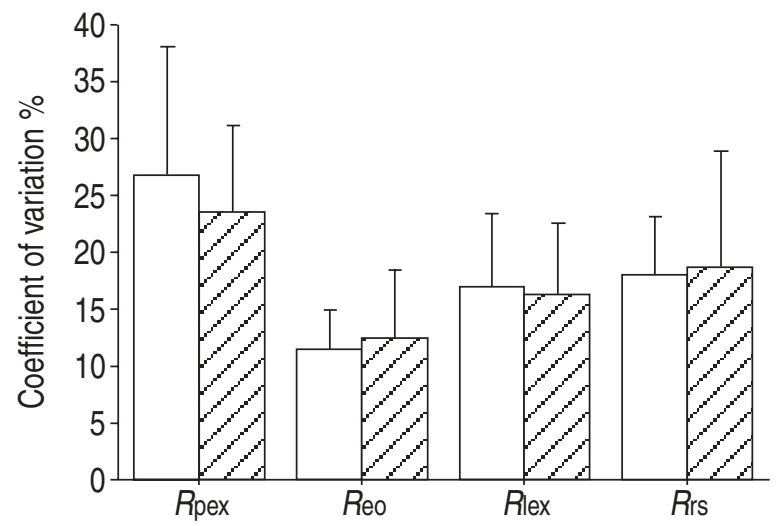

Fig. 3. - The coefficient of variation $(\mathrm{CV})$ of interrupter resistance (Rint) data were significantly dependant upon the analysis technique used (Two way ANOVA: $\mathrm{p}<0.001)$. $R_{\text {pex }}(R$ int determined using polynomial back extrapolation method) and $R$ lex (Rint determined using linear back extrapolation method) were significantly more and less variable, respectively when compared to $R$ eo (instantaneous $R$ int following the end of pressure oscillations) and respiration system resistance $(R \mathrm{rs})$ (Pairwise comparisons: $\mathrm{p}<0.05)$. The mask type did not significantly influence the CV of $R$ int data. Columns ( $\square$ : Mcomp; $\mathbb{Z}$ : Mrigid) and error bars represent mean and $\mathrm{SD}$, respectively.

facemask (fig. 2). Rpex and $R$ lex were not significantly different from each other $\left(R\right.$ pex: $3.86 \pm 0.2 \mathrm{kPa} \cdot \mathrm{L} \cdot \mathrm{s}^{-1}$, $R$ lex: $\left.3.84 \pm 0.2 \mathrm{kPa} \cdot \mathrm{L} \cdot \mathrm{s}^{-1}\right)$, however, both were significantly lower than $R$ eo $\left(5.06 \pm 0.2 \mathrm{kPa} \cdot \mathrm{L} \cdot \mathrm{s}^{-1} ; \mathrm{p}<0.05\right)$. $R$ rs was significantly higher than $R$ int values calculated from the remaining analysis techniques with a group mean of $16.88 \pm 1.25 \mathrm{kPa} \cdot \mathrm{L} \cdot \mathrm{s}^{-1} \quad(\mathrm{p}<0.05)$. Individual Rint data for Mcomp and Mrigid are shown in table 1. The CV was significantly influenced by the choice of analysis technique (fig. 3). Group mean CV was lowest for Reo $(11.9 \pm 1.4 \% ; \mathrm{p}<0.05)$ and highest for $R$ pex $(25.1 \pm 1.4 \% ; \mathrm{p}<0.05)$, while Rrs $(18.4 \pm 1.4 \%)$

Table 1. - Individual subject interrupter resistance data

\begin{tabular}{|c|c|c|c|c|c|c|c|c|}
\hline \multirow[t]{2}{*}{ Subject } & \multicolumn{2}{|c|}{$R$ pex } & \multicolumn{2}{|c|}{$R \operatorname{lex}$} & \multicolumn{2}{|c|}{ Reo } & \multicolumn{2}{|c|}{ Rrs } \\
\hline & Mcomp & Mrigid & Mcomp & Mrigid & Mcomp & Mrigid & Mcomp & Mrigid \\
\hline 1 & $1.87 \pm 0.95$ & $6.91 \pm 0.81$ & $2.16 \pm 0.59$ & $6.81 \pm 0.89$ & $3.63 \pm 0.54$ & $7.51 \pm 1.02$ & $17.25 \pm 3.15$ & $13.81 \pm 2.64$ \\
\hline 2 & $2.58 \pm 0.94$ & $4.11 \pm 0.75$ & $2.78 \pm 0.55$ & $3.85 \pm 0.21$ & $4.02 \pm 0.56$ & $4.68 \pm 0.35$ & $16.16 \pm .3 .36$ & $10.65 \pm 4.54$ \\
\hline 3 & $1.93 \pm 0.63$ & $2.30 \pm 0.82$ & $2.30 \pm 0.35$ & $2.54 \pm 0.48$ & $3.05 \pm 0.26$ & $3.60 \pm 0.44$ & $10.06 \pm 2.42$ & $13.63 \pm 1.10$ \\
\hline 4 & $2.19 \pm 0.60$ & $3.92 \pm 1.34$ & $1.92 \pm 0.52$ & $3.98 \pm 1.08$ & $2.90 \pm 0.33$ & $5.17 \pm 1.25$ & $13.98 \pm 1.82$ & $15.38 \pm 5.71$ \\
\hline 5 & $3.57 \pm 0.73$ & $3.78 \pm 0.72$ & $3.08 \pm 0.34$ & $3.51 \pm 0.56$ & $4.14 \pm 0.47$ & $4.79 \pm 0.50$ & $12.96 \pm 2.76$ & $20.04 \pm 3.58$ \\
\hline 6 & $4.55 \pm 0.68$ & $5.42 \pm 0.98$ & $4.42 \pm 0.48$ & $5.43 \pm 0.59$ & $5.73 \pm 0.69$ & $6.77 \pm 0.70$ & $16.02 \pm 2.40$ & $18.91 \pm 2.58$ \\
\hline 7 & $3.64 \pm 0.55$ & $5.32 \pm 0.99$ & $3.44 \pm 0.34$ & $5.18 \pm 0.61$ & $4.55 \pm 0.52$ & $6.32 \pm 0.66$ & $14.23 \pm 3.22$ & $19.13 \pm 3.74$ \\
\hline 8 & $2.94 \pm 0.22$ & $5.67 \pm 1.77$ & $2.67 \pm 0.28$ & $5.76 \pm 1.07$ & $3.25 \pm 0.13$ & $7.28 \pm 0.98$ & $7.82 \pm 0.80$ & $24.41 \pm 2.36$ \\
\hline 9 & $2.42 \pm 0.79$ & $4.47 \pm 1.04$ & $3.08 \pm 0.58$ & $4.92 \pm 0.96$ & $5.13 \pm 0.75$ & $7.03 \pm 1.08$ & $25.79 \pm 5.03$ & $27.63 \pm 4.17$ \\
\hline 10 & $2.86 \pm 0.98$ & $4.44 \pm 0.89$ & $2.60 \pm 0.29$ & $4.40 \pm 0.59$ & $3.36 \pm 0.41$ & $6.26 \pm 0.33$ & $18.42 \pm 2.82$ & $21.71 \pm 3.45$ \\
\hline 11 & $2.27 \pm 0.40$ & $4.44 \pm 0.75$ & $1.89 \pm 0.30$ & $4.40 \pm 0.55$ & $3.01 \pm 0.20$ & $6.26 \pm 0.43$ & $18.77 \pm 1.76$ & $21.71 \pm 1.29$ \\
\hline 12 & $3.78 \pm 1.23$ & $6.03 \pm 1.83$ & $3.44 \pm 0.95$ & $5.96 \pm 1.48$ & $4.65 \pm 0.75$ & $7.01 \pm 1.71$ & $15.93 \pm 3.09$ & $19.42 \pm 4.31$ \\
\hline 13 & $4.85 \pm 1.36$ & $4.19 \pm 1.34$ & $5.20 \pm 0.78$ & $4.52 \pm 1.13$ & $6.52 \pm 0.91$ & $5.89 \pm 0.87$ & $20.35 \pm 5.52$ & $20.52 \pm 3.38$ \\
\hline 14 & $3.28 \pm 0.79$ & $4.44 \pm 0.85$ & $3.11 \pm 0.50$ & $4.20 \pm 0.45$ & $3.93 \pm 0.41$ & $5.22 \pm 0.30$ & $11.24 \pm 1.90$ & $12.92 \pm 2.44$ \\
\hline Mean \pm SEM & $3.05 \pm 0.25$ & $4.67 \pm 0.30$ & $3.00 \pm 0.25$ & $4.68 \pm 0.29$ & $4.13 \pm 0.29$ & $5.98 \pm 0.31$ & $15.64 \pm 1.21$ & $18.56 \pm 1.27$ \\
\hline
\end{tabular}

Individual interrupter resistance $(R \mathrm{int})(\operatorname{mean} \pm \mathrm{SD})$ data for the four analysis techniques following the application of the large volume, compliant facemask (Mcomp) and a small volume, rigid facemask (Mrigid). The analysis techniques were the: polynomial back extrapolation technique $(R$ pex $)$, linear back extrapolation technique $(R$ lex $)$, the mean instantaneous airway opening pressure $(P \mathrm{ao})$ after the pressure oscillations had ended (Reo) and the mean $P$ ao at the end of the occlusion (Rrs). 
and $R$ lex $(16.6 \pm 1.4 \%)$ were not different from each other.

\section{Discussion}

The primary purpose of this investigation was to evaluate the feasibility of using the interrupter technique in spontaneously breathing infants. This involved determining a suitable $V^{\prime}$ int, $t$ int for infant studies and evaluating the influence of differing facemasks and analysis techniques on the magnitude and variability of $P$ post and hence $R$ int. It could be demonstrated that the interrupter technique is feasible in healthy, unsedated infants. The choice of facemask plays a crucial role, with Mcomp causing a significant underestimation of Rint when compared to Mrigid. The method of determining $P$ post also significantly influenced the variability and magnitude of $R$ int value.

\section{Factors influencing suitable triggering}

The most suitable $V^{\prime}$ int may be altered in infants, who have higher respiratory rates and lower tidal flows and volumes when compared to older children. The most appropriate trigger would be a stable volume landmark, thus ensuring a consistent lung volume during interruptions and minimizing the volume dependant effects of $R$ int. The disadvantage of a volume related trigger is the identification of a stable landmark, particularly in infants. The use of flow as a trigger reduces software and hardware delays as $V^{\prime}$ is directly measured while the $V$ is calculated. The choice of $20 \mathrm{~mL} \cdot \mathrm{s}^{-1}$ for $V^{\prime}$ int produced occlusion volumes equating to $90.7 \pm 1.6 \%$ and $92.5 \pm 0.4 \%$ of the inspiratory volume for Mcomp and Mrigid, respectively. Indicating that reproducible lung volumes are feasible with a suitable $V^{\prime}$ int. PEF ranged between 33.1 and $73.3 \mathrm{~mL} \cdot \mathrm{s}^{-1}$ in this study group clearly indicating that $V^{\prime}$ int must be tailored to patient group to be tested. Thus the standardization of a specific triggering flow may not be possible, rather future studies should report the trigger flow, PEF and absolute and relative occlusion volumes to allow comparisons between study centres.

\section{Influence of the facemask on interrupter resistance}

Previous studies have demonstrated that the addition of a proximal compliance caused an underestimation of the Ppost [7, 8]. Simulating an unsupported airway, SLY et al. [7] reported an underestimation of Rint of up to $75.8 \%$, which was reduced to a maximum error of $15.2 \%$ with a support airway. In older healthy and asthmatic children supporting the cheeks increased $R$ int by 20.5 and $32.7 \%$, respectively [8]. These results suggest that as obstruction increases that the error introduced by a proximal compliance increases proportionally. The data in the present study confirm that increasing the proximal compliance (Mcomp) causes an underestimation of Rint in infants. The use of Mrigid increased Rint by values ranging from $18.7 \%(R$ rs $)$ to $55.7 \%$ (Rlex). These data illustrate the importance of minimizing the proximal compliance, indicating that the correct choice of facemask is an important factor in infant lung function studies. The use of a rigid walled mask will minimize the dead space and reduce compliance of the upper airways and measurement equipment.

\section{Factors affecting the estimation of interrupter resistance}

The main assumption of the interrupter technique is that $P$ ao rapidly equilibrates with $P$ alv during a brief airway closure. Lung inhomogeneities and Cua will influence the time for equalization of $P$ ao and $P$ alv. In older children and adults an interruption time of $80-100 \mathrm{~ms}$ is commonly used. The study of lung function in infants requires the use of a facemask placed over the nose and mouth. Thus the measured resistance of the airway tree will include the nasal pathway, which is known to contribute up to $49 \%$ of the measured airway resistance [18]. This additional resistance will significantly increase the time constant of the respiratory system and hence the occlusion time required to allow accurate confirmation of a relaxed passive system. In infants, the presence of the HeringBreuer reflex allows longer occlusion times to be used and thus ensure that $P$ alv equilibrates $P$ ao, even in the presence of airway obstruction. The present study used an occlusion time of $500 \mathrm{~ms}$, based on the occlusion time recommended for the single occlusion technique in infants [12]. Interruptions of this length were achievable in all infants without disturbing their sleeping patterns. The additional benefit of a longer occlusion time is that $R$ int calculated from $P$ ao at the end of the interruption equates to $R$ rs, allowing estimates of both airway and respiratory resistances to be obtained. The method used to determine $P$ post had an important effect on the magnitude and variability of $R$ int. Three of the analysis methods used allow the estimation of the resistance of the airway tree, namely $R$ pex, $R$ lex and $R$ eo, while $R$ int calculated from the end of the interruption ( $R \mathrm{rs})$ equates to the resistance of the airways and respiratory tissues, including the chest wall. $R$ pex and $R$ lex were not significantly different from each other and were both significantly lower than Reo. This is not unexpected as $R$ eo is estimated from the instantaneous $P$ ao at a fixed time point following the pressure oscillations, whereas Rpex and Rlex are determined from the back extrapolation of the $P$ ao trace and thus derived from an earlier time point and hence lower $P$ ao. Reo may overestimate $R$ int under conditions in which the concave shape of the $P$ ao trace becomes more pronounced (i.e. airway obstruction), the value of $R$ eo is also highly dependent on the time point chosen. The characteristics of the pressure oscillations are determined by the inertia and compressibility of the gas column [19, 20]. Alterations in the inertance and compliance of the airway wall will lead to changes in the frequency and damping of the pressure oscillations and may result in the time point chosen being invalid. The values of $R$ rs reported in the present 
study are significantly higher than those reported in the literature. In a large cohort of infants (aged 2-10 weeks, group mean 5 weeks) studied prior to incidence of respiratory illness, Young et al. [21] reported mean $R$ rs values of $5.6 \pm 0.1 \mathrm{kPa} \cdot \mathrm{L} \cdot \mathrm{s}^{-1}$ (mean \pm SEM) using the single breath technique. Using the same technique, Dundas et al. [22] reported similar values of $5.3 \pm 1.7 \mathrm{kPa} \cdot \mathrm{L} \cdot \mathrm{s}^{-1}$ (mean $\left.\pm \mathrm{SD}\right)$ in healthy infants less than 13 weeks of age. The reasons for the differences between these previously reported values of $R \mathrm{rs}$ and those in the present study $(18.6 \pm 1.3 ; 10.7-$ $27.6 \mathrm{kPa} \cdot \mathrm{L} \cdot \mathrm{s}^{-1}$ (mean $\pm \mathrm{SEM}$; range)) using Mrigid are not clear. The infants in the present study were unsedated, whereas previous investigations used sedation. Sedation is known to influence lung volumes and hence may influence lung mechanics. As far as the authors are aware there are no published data for the interrupter technique in young, unsedated infants and hence it is difficult to meaningfully compare the data with those $R$ rs data published with differing methodologies. However, in a group of healthy infants aged between 4.2 and 8 weeks RABBETTE et al. [23] reported end-inspiratory elastic recoil pressures ranging $0.78-1.02 \mathrm{kPa}$, which compares favourably to the end interruption $P$ ao determined with Mrigid in the present study $(0.59-1.06 \mathrm{kPa})$.

In agreement with previous studies in children and adults $R$ pex was significantly more variable than the remaining estimation techniques $[2,10]$. Furthermore, as reported by PHAGOo et al. [2] in five-year-old children, Rlex was the least variable. As the subjects in the present study were healthy, unsedated infants it was not possible to carry out challenge testing for ethical reasons, and as such no direct conclusions as to the most sensitive and appropriate interrupter analysis method for challenge testing can be made.

In summary aspects of measurement set-up and subsequent analysis influence the accuracy, technical feasibility and variability of the interrupter technique when applied to an infant population were examined. The study demonstrated that the interrupter technique is feasible in spontaneously breathing, sleeping infants. The ability to apply the interrupter technique to an unsedated, sleeping infant population could potentially allow the technique to be used for large epidemiological studies. Also of importance may be the application of the technique for the clinical monitoring of infants with asthma and other respiratory diseases. Occlusions of $500 \mathrm{~ms}$ could be applied with no respiratory muscle activity present in the majority of interruptions. The choice of facemask in this age group significantly influenced the subsequent Rint values and future studies should use rigid wall, noncompliant masks. The reduction of dead space through the use of suitable putty would also be desirable. It was demonstrated that the linear extrapolation method was the least variable analysis technique. Future use of the interrupter technique will need to report factors that are known to influence the outcome of Rint studies, including facemask type and dead space, the analysis technique, occlusion length and the flow at which the occlusion is triggered. Ideally these factors should be standardized, at least within specified age ranges, allowing reference data to be generated that may be used by a number of study centres. Challenge testing in infants of this age remains to be carried out to confirm the clinical usefulness of the interrupter technique in spontaneously breathing infants.

Acknowledgements. The authors would like to thank H. Staub for help in recruiting the subjects and collecting the study data.

\section{References}

1. Klug B, Bisgaard $\mathrm{H}$. Measurement of lung function in awake 2-4-year-old asthmatic children during methacholine challenge and acute asthma: a comparison of the impulse oscillation technique, the interrupter technique, and transcutaneous measurement of oxygen versus whole-body plethysmography. Pediatr Pulmonol 1996; 21: 290-300.

2. Phagoo SB, Wilson NM, Silverman M. Evaluation of the interrupter technique for measuring change in airway resistance in 5-year-old asthmatic children. Pediatr Pulmonol 1995; 20: 387-395.

3. Phagoo SB, Wilson NM, Silverman M. Evaluation of a new interrupter device for measuring bronchial responsiveness and the response to bronchodilator in 3 year old children. Eur Respir J 1996; 9: 1374-1380.

4. Bisgaard H, Klug B. Lung function measurement in awake young children. Eur Respir J 1995; 8: 20672075.

5. Ludwig MS, Romero PV, Sly PD, Fredberg JJ, Bates JH. Interpretation of interrupter resistance after histamine-induced constriction in the dog. J Appl Physiol 1990; 68: 1651-1656.

6. Bates JH, Ludwig MS, Sly PD, Brown K, Martin JG, Fredberg JJ. Interrupter resistance elucidated by alveolar pressure measurement in open-chest normal dogs. J Appl Physiol 1988; 65: 408-414.

7. Sly PD, Bates JH. Computer analysis of physical factors affecting the use of the interrupter technique in infants. Pediatr Pulmonol 1988; 4: 219-224.

8. Oswald-Mammosser M, Llerena C, Speich JP, Donata L, Lonsdorfer J. Measurements of respiratory system resistance by the interrupter technique in healthy and asthmatic children. Pediatr Pulmonol 1997; 24: 78-85.

9. Bates JH, Sly PD, Kochi T, Martin JG. The effect of a proximal compliance on interrupter measurements of resistance. Respir Physiol 1987; 70: 301-312.

10. Phagoo SB, Watson RA, Pride NB, Silverman M. Accuracy and sensitivity of the interrupter technique for measuring the response to bronchial challenge in normal subjects. Eur Respir J 1993; 6: 996-1003.

11. Klug B, Bisgaard H. Specific airway resistance, interrupter resistance, and respiratory impedance in healthy children aged 2-7 years. Pediatr Pulmonol 1998; 25: 322-331.

12. Fletcher ME, Baraldi E, Steinbrugger B. Passive respiratory mechanics. In: Stocks J, Sly PD, Tepper RS, Morgan WJ, eds. Infant Lung Function testing. New York, Wiley-Liss, 1996; pp. 283-327.

13. Otis AB, Proctor DF. Measurement of alveolar pressure in human subjects. Am J Physiol 1948; 152: 106-112. 
14. Chowienczyk PJ, Lawson CP, Lane S, et al. A flow interruption device for measurement of airway resistance. Eur Respir J 1991; 4: 623-628.

15. Bates JH, Sly PD, Okubo S. General method for describing and extrapolating monotonic transients and its application to respiratory mechanics. Med Biol Eng \& Comput 1987; 25: 131-135.

16. Fichter J, Wierich W, Hartung W. Resistance measurement in normal and obstructed excised human lungs by means of the interrupter method. Respiration 1989; 56: $34-42$.

17. Frey U, Stocks J, Coates A, Sly PD, Bates JHT. Specifications for equipment used for infants pulmonary testing. Eur Respir J 2000; 16: 731-740.

18. Stocks J, Godfrey S. Nasal resistance during infancy. Respir Physiol 1978; 34: 233-246.

19. Frey U, Schibler A, Kraemer R. Pressure oscillations after flow interruption in relation to lung mechanics. Respir Physiol 1995; 102: 225-237.

20. Shephard RJ. Mechanical characteristics of the human airway in relation to use of the interrupter valve. Clin Sci 1962; 25: 263-280.

21. Young S, O'Keeffe PT, Arnott J, Landau LI. Lung function, airway responsiveness, and respiratory symptoms before and after bronchiolitis. Arch Dis Child 1995; 72: 16-24.

22. Dundas I, Dezateux CA, Fletcher ME, Jackson EA, Stocks J. Comparison of single-breath and plethysmographic measurements of resistance in infancy. $\mathrm{Am}$ J Respir Crit Care Med 1995; 151: 1451-1458.

23. Rabbette PS, Fletcher ME, Dezateux CA, SorianoBrucher H, Stocks J. Hering-Breuer reflex and respiratory system compliance in the first year of life: a longitudinal study. J Appl Physiol 1994; 76: 650-656. 